\title{
Preparation and evaluation of chitosan-polyvinyl alcohol/ polyhexamethylene guanidine hydrochloride antibacterial dressing to accelerate wound healing for infectious skin repair
}

\author{
Xiaotong Yue ${ }^{1}$, Lingying Liu ${ }^{1}$, Yushou $\mathrm{Wu}^{1}$, Xiangyu Liu ${ }^{1}$, Shaozeng $\mathrm{Li}^{2}$, Zihao Zhang ${ }^{1}$, Shaofang Han ${ }^{1}$, \\ Xiaoteng Wang', Yang Chang ${ }^{1}$, Hailiang Bai ${ }^{1}$, Jiake Chai ${ }^{1}$, Sen $\mathrm{Hu}^{3}$, Hongyu Wang ${ }^{4}$ \\ ${ }^{1}$ Burns Institute of PLA, Department of Burns and Plastic Surgery, the Fourth Medical Center Affiliated to PLA General Hospital, Beijing, China; \\ ${ }^{2}$ Department of Clinical Laboratory, the Fourth Medical Center Affiliated to PLA General Hospital; Beijing, China; ${ }^{3}$ Research Laboratory of Trauma \\ Surgery, the Fourth Medical Center Affiliated to PLA General Hospital, Beijing, China; ${ }^{4}$ Department of Burn Surgery, the Third Affiliated Hospital \\ of Inner Mongolia Medical University (Inner Mongolia Baogang Hospital), Baotou, China \\ Contributions: (I) Conception and design: X Yue, L Liu; (II) Administrative support: Medical School of Chinese PLA; (III) Provision of study materials \\ or patients: X Yue, L Liu, Y Wu; (IV) Collection and assembly of data: H Wang; (V) Data analysis and interpretation: X Yue; (VI) Manuscript \\ writing: All authors; (VII) Final approval of manuscript: All authors. \\ Correspondence to: Jiake Chai. Department of Burn \& Plastic Surgery, the Fourth Medical Center Affiliated to PLA General Hospital, No. 51 Fucheng \\ Road, Haidian District, Beijing 100048, China. Email: cjk304@126.com.
}

Background: Wound infections, especially multidrug-resistant (MDR) bacterial infections, are a major challenge in clinical medicine.

Methods: In this study, a new type of antibacterial sponge was prepared from a solution containing a chitosan-polyvinyl alcohol (CTS-PVA) emulsion with added polyhexamethylene guanidine hydrochloride (PHMG) in a homogeneous medium using lyophilization technology. The antibacterial ability of and CTSPVA/PHMG sponge against Staphylococcus aureus, Pseudomonas aeruginosa, Escherichia coli, Candida albicans, Methicillin-resistant Staphylococcus aureus, multidrug-resistant Pseudomonas aeruginosa, and multidrug-resistant Acinetobacter baumannii in vitro. The structure and physical properties were characterized. The sponge dressing was tested in a Pseudomonas aeruginosa-infected full-thickness mouse skin wound defect model. The effects were evaluated by wound area measurement and histological analysis.

Results: The CTS-PVA/PHMG sponge showed broad-spectrum antibacterial ability, including for MDR bacterial stains from clinical sources, while maintaining excellent physicochemical properties, including a high swelling degree and good moisture retention capability. Scanning electron microscopy images displayed the surface morphology of the CTS-PVA/PHMG sponge dressing. The detection of the wound healing rate and histological analysis supported that the new dressing can alleviate the inflammation and accelerate the healing speed of infected wounds and in vivo.

Conclusions: CTS-PVA/PHMG sponge shows broad-spectrum antibacterial activity, which can provide a new pathway for clinical prevention and treatment of superbug-infected wounds.

Keywords: Antibacterial; infectious wound; polymer; dressing

Submitted Jan 12, 2021. Accepted for publication Mar 16, 2021.

doi: $10.21037 / \mathrm{atm}-21-509$

View this article at: http://dx.doi.org/10.21037/atm-21-509

(c) Annals of Translational Medicine. All rights reserved. 


\section{Introduction}

Wound infections have high rates of morbidity and associated mortality (1). Almost 14 million people per year in the United States are affected by wound infections $(2,3)$, ranging from minor superficial to life-threatening infections (4). In recent decades, with the abuse of antibiotics, more than $70 \%$ of wound bacteria have been resistant to at least 1 of the commonly used antibiotics (5). Laboratories around the world are committed to developing antibacterial dressings to prevent wound infections, improve the healing process, and reduce the mortality rate of multiresistant bacterial strains and incurable wound infections. Different agents, including antibiotics, nanoparticles [e.g., silver nanoparticles (AgNP)] and polymers [e.g., chitosan (CTS)], have been incorporated within wound dressings to improve antimicrobial activity (6-11).

Guanidine polymers are polycationic antimicrobial polymers with strong antibacterial properties. Bacteria and fungi are generally negatively charged on the surface due to the proteins or liquids in their cell membrane. Polycations are attracted by electrostatic forces to bacterial or fungal cells and are able to disrupt the outer membrane as well as the cytoplasmic membrane and to achieve cell lysis, resulting in cell death (12). Guanidinium-rich polymers do not produce specific binding with the bacterial cell membrane but achieve the ultimate sterilization purpose via electrostatic attraction to the cell membrane; thus, they do not easily trigger drug resistance. Since the outer membrane of mammalian cells is electrically neutral, the positively charged guanidine polymer has strong selectivity for bacterial or fungal cell membranes (13). Compared with the amine group, the guanidine group binds more firmly to the cell membrane and has more powerful antibacterial properties.

Commercial Prontosan ${ }^{\circledR}$ liquid guanidine dressings apply small molecular guanidine salts directly to wounds. Sun grafted polyhexamethylene guanidine hydrochloride (PHMG) onto the polymer side chain to prevent the leakage of antibacterial agents and increase the antibacterial durability of the dressing (14). However, this preparation process has toxic solvent residues, so it is not suitable for clinical application in wound repair.

To overcome the shortcomings of the above antibacterial dressings, CTS and polyvinyl alcohol (PVA) were used as polymeric materials to prepare composites incorporating the polycationic polymer PHMG to obtain a new type of antibacterial dressing in the present study. The sponges were characterized by scanning electron microscopy, swelling rate, and antibacterial analyses in vivo and in vitro.
We present the following article in accordance with the ARRIVE reporting checklist (available at http://dx.doi. org/10.21037/atm-21-509).

\section{Methods}

\section{Materials}

We purchased PHMG from Hipoly Co, Ltd, (Shanghai, China). The PVA (with a degree of polymerization of $\sim 1,700$ and a degree of alcoholysis of 99\%) was purchased from Guoyao Co, Ltd, (Zhejiang, China). The CTS (with a degree of deacetylation $\geq 95 \%$ ) was purchased from Qingdao Haihui Biotechnology Co, Ltd, (Shandong, China). Staphylococcus aureus (S. aureus, gram-positive bacteria, ATCC 25923), Pseudomonas aeruginosa (P. aeruginosa, gramnegative bacteria, ATCC 27853), Escherichia coli (E. coli, gram-negative bacteria, ATCC25922), and Candida albicans (C. albicans, fungus, ATCC 90028) were obtained from the Fourth Medical Center Affiliated to PLA General Hospital. Methicillin-resistant Staphylococcus aureus (MRSA), multidrug-resistant Pseudomonas aeruginosa (MDR-PA), and multidrug-resistant Acinetobacter baumannii (MDR$\mathrm{AB})$ were obtained from the participants with their consent. One hundred and twenty the specific pathogen-free (SPF) male BALB/c mice ( 8 weeks with bodyweight between 20-25 g) were purchased by Beijing Keyu Animal Aquacluture Center (Beijing, China).The animals were housed at room temperature $\left(25^{\circ} \mathrm{C}\right)$ in the animal room (SPF class) of the Fourth Medical Center Affiliated to PLA General Hospital. This study was performed in accordance with the Guide for Care and Use of Laboratory Animals of National Research Council and approved by the Fourth Medical Center Affiliated to PLA General Hospital, Beijing, China.

\section{The antibacterial activity of PHMG solution}

Antibacterial activity of the PHMG solution was tested via the microtiter broth dilution method, which determined the minimum inhibitory concentration (MIC) that led to the inhibition of microbial growth. The antibacterial efficacies of PHMG were evaluated for both laboratory antibioticsensitive strains (gram-positive bacteria $S$. aureus, gramnegative bacteria $E$. coli and $P$. aeruginosa, fungi $C$. albicans) and clinical MRSA, MDR-PA, and MDR-AB. First, $1 \mathrm{~mL}$ of bacterial suspension $\left(1 \times 10^{6} \mathrm{CFU} / \mathrm{mL}\right)$ was added to each sterile colorimetric test tube. Then, $1 \mathrm{~mL}$ of the PHMG solutions of different concentrations with Luria-Bertani 


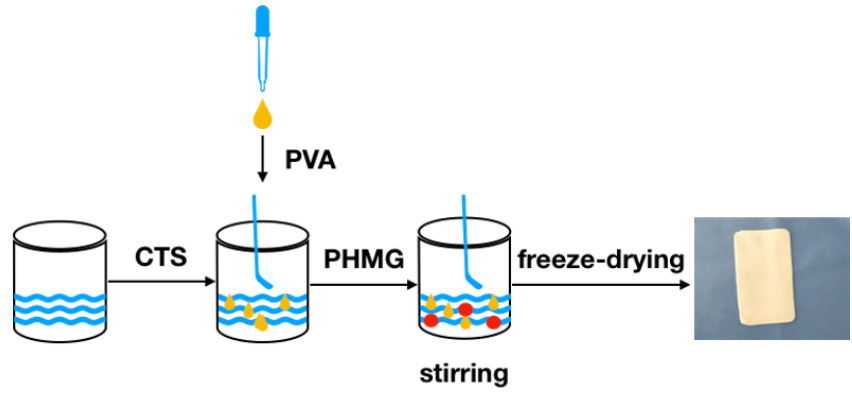

Figure 1 Preparation process of the CTS-PVA sponge. CTS, chitosan; PVA, polyvinyl alcohol.

(LB) medium were added to each test tube so that the final bacterial concentration in the test tube was $5 \times 10^{5} \mathrm{CFU} / \mathrm{mL}$, and the PHMG concentration was $0 \%, 0.05 \%, 0.1 \%$, $0.2 \%, 0.4 \%, 1 \%, 2 \%$, and $4 \%$. An LB medium without the addition of bacteria was used as the control group. The test tube rack was placed in a bacterial incubator for $24 \mathrm{~h}$. The minimum liquid concentration in the clarified test tube was the minimum inhibitory concentration (MIC) of the PHMG solution (15).

\section{Preparation of the CTS-PVA/PHMG dressing}

We added $2 \mathrm{~g}$ of CTS to $100 \mathrm{~mL}$ of $1 \%$ acetic acid and it was magnetically stirred at $1,500 \mathrm{rpm}$ to obtain a $2 \%$ CTS solution. A total of $5 \mathrm{~g}$ of PVA was dissolved in ultrapure water (UPW) for $2 \mathrm{~h}$ at $80^{\circ} \mathrm{C}$ to obtain a $5 \%$ PVA solution. Then, $5 \mathrm{~mL}$ of the PVA solution was added to $100 \mathrm{~mL}$ of the CTS solution and stirred mechanically for $5 \mathrm{~min}$ at 2,000 rpm. The PHMG solution was separately added to final solution at concentrations of $0.05 \%, 0.1 \%$, $0.2 \%$, and $0.4 \%$ wt. The CTS-PVA/PHMG composite was poured into a metal container with a bottom surface of $100 \mathrm{~mm} \times 200 \mathrm{~mm}$ at $4{ }^{\circ} \mathrm{C}$ for $30 \mathrm{~min}$ and then placed at $-20{ }^{\circ} \mathrm{C}$ for $2 \mathrm{~h}$, followed by $-80{ }^{\circ} \mathrm{C}$ for $12 \mathrm{~h}$. Finally, the solution was freeze-dried using a vacuum freezer drier for $48 \mathrm{~h}$, resulting in CTS-PVA/PHMG dressings with different PHMG concentrations (denoted CTS-PVA/ PHMG0.05, CTS-PVA/PHMG0.1 and CTS-PVA/ PHMG0.2, CTS-PVA/PHMG0.4, respectively) (Figure 1).

\section{In vitro antibacterial ability test of the CTS-PVA/PHMG dressing}

The antibacterial activities of CTS-PVA/PHMG were evaluated by determining the optical density (OD) (600 nm) of the bacterial suspensions co-incubated with different PHMG concentrations for $24 \mathrm{~h}$; the untreated bacterial suspensions were used as a control. Composite CTS-PVA/ PHMG samples $(1 \mathrm{~cm} \times 1 \mathrm{~cm})$ with various concentrations were placed in a 48 -well plate. Then, $500 \mu \mathrm{L}$ of bacterial solution $\left(5 \times 10^{5} / \mathrm{mL}\right)$ with $\mathrm{LB}$ medium was added to the 48 -well plate and placed in a $37^{\circ} \mathrm{C}$ incubator for $24 \mathrm{~h}$.

A total of $100 \mu \mathrm{L}$ bacterial solution was taken from each well of a 96-well plate, and the absorbance was measured on a microplate reader at $600 \mathrm{~nm}$. Then, $100 \mu \mathrm{L}$ of the bacterial suspension was pipetted onto the surface of a Mueller Hinton ( $\mathrm{MH}$ ) plate, spread uniformly by a coating rod, and the bacteria were incubated overnight for $24 \mathrm{~h}$ at $37^{\circ} \mathrm{C}$. The bacterial colonies grown on the $\mathrm{MH}$ plate were counted, and the minimal bactericidal concentration [(MBC) less than 5 colonies] of each sample was obtained after MBC tests. All samples were repeated in triplicate (16).

\section{Characterization}

SEM images of the CTS-PVA/PHMG sponge dressing The microstructures of the CTS-CTA, CTS-PVA/ PHMG0.05, CTS-PVA/PHMG0.1, CTS-PVA/PHMG0.2, and CTS-PVA/PHMG0.4 sponge dressings were examined by scanning electron microscopy (SEM, Hitachi Co, Ltd, Tokyo, Japan; operating at $10 \mathrm{kV}$ ).

\section{Degree of swelling and moisture-retention capacity}

The sponge dressings were immersed in UPW, phosphatebuffered saline (PBS), simulated body fluid (SBF), and fetal bovine serum (FBS) for $24 \mathrm{~h}$ at room temperature until swelling equilibrium was reached. The degree of swelling was calculated as follows:

$$
\text { Degree of swelling }=\frac{m 2-m 1}{m 1} \times 100 \%
$$

In this equation, $\mathrm{m} 1$ and $\mathrm{m} 2$ are the weights of the dressings before and after immersion.

To measure the moisture retention of the dressing, the wet dressing fully engorged with UPW was placed in a glass dryer at room temperature, and the degree of swelling was measured every $2 \mathrm{~h}$. All samples were tested in triplicate (17).

\section{Infectious wound of full-thickness skin defect in mice}

\section{Macroscopic appearances}

The BALB/c male mice $(\mathrm{n}=120)$ were randomly assigned to 4 groups (Gauze, Ag, CTS-PVA, CTS-PVA/PHMG), with 30 mice in each group. The mice were successfully 
anaesthetized by intraperitoneal injection of pentobarbital $(60 \mathrm{mg} / \mathrm{kg})$. A full-thickness wound model $(10 \mathrm{~mm}$ diameter) was used on the back after the fur was shaved. Wounds were inoculated with P. aeruginosa $(100 \mu \mathrm{L}$, $10^{8} \mathrm{CFU} / \mathrm{mL}$ ) for $30 \mathrm{~min}$, and infected wound models were established. The wounds were treated with Gauze, silver (Ag), CTS-PVA, and CTS-PVA/PHMG dressings (size: $20 \mathrm{~mm} \times 20 \mathrm{~mm}$ ). Finally, Urgo strapping restraining bandages (URGO, Paris, France) were used to fix the wound and dressings. The wound dressings in each group were changed every 3 days according to the above methods.

\section{Wound healing rate}

The wound areas were photographed and measured on the $3 \mathrm{rd}, 7$ th, and 14th days after the operation. All wounds areas were measured using Image-Pro Plus 6.0 software (Media Cybernetics Corp, Rockville, MD, USA), and the wound closure rates were then calculated as follows:

$$
\text { Wound closure rate }=\frac{A o-A t}{A o} \times 100 \%
$$

$A 0$ is the initial wound area, and $A t$ is the wound area on the $3 \mathrm{rd}, 7 \mathrm{th}, 10 \mathrm{th}$, and 14 th days after the operation.

\section{Histological analysis}

The wound skins were collected on the $3 \mathrm{rd}, 7 \mathrm{th}, 10 \mathrm{th}$, and 14th days after the operation and fixed in $4 \%$ polyformaldehyde solution. The histopathological sections were stained with hematoxylin and eosin (H\&E). Histological images were taken by an inverted microscope.

\section{Statistical analysis}

All values were expressed as the mean \pm standard deviation. One-way analysis of variance (ANOVA) was applied to compare the differences between groups. Pairwise comparisons among the means were conducted by the Least Significant Difference (LSD) method. A P value less than 0.05 was considered statistically significant.

\section{Results}

\section{Antibacterial activity of the PHMG solution}

The PHMG $0 \%$ group of the mixture became turbid, suggesting that the strains rapidly proliferated. The control group was pellucid, which meant that the suspension was free of pollution. When the PHMG concentration was $0.05 \%$, the medium was slightly turbid compared with the control group, indicating that few strains proliferated. When the PHMG concentration was $0.1 \%, 0.2 \%, 0.4 \%, 1 \%$, and $4 \%$, the clarity was the same as that of the control group, which was the minimum inhibitory concentration. All the above strains were inhibited by PHMG with an MIC of $0.1 \%$. According to the inhibitory concentration results, PHMG at $0.05 \%, 0.1 \%$, $0.2 \%$, and $0.4 \%$ was screened out for subsequent preparation of antibacterial sponge dressings (see Figure 2).

\section{The antibacterial ability of CTS-PVA sponge dressings loaded with PHMG in vitro}

The CTS-PVA group had a certain decrease in the absorbance of $P$. aeruginosa and MDR-PA relative to the untreated bacterial suspensions, but the OD value was still significantly higher than that of the control group $(\mathrm{P}<0.01)$. The OD values of the CTS-PVA group for $E$. coli and $C$. albicans were significantly lower than those of the bacterial suspensions. The OD values of CTS/PVA-PHMG0.05, CTS-PVA/PHMG0.1, CTS-PVA/PHMG0.2, and CTSPVA/PHMG0.4 were not significantly different from that of the control group $(\mathrm{P}>0.05)$ (see Figure $3 A)$.

After the seven suspensions were coated on the plate, the colonies grew over the entire MH plate. Compared with the control group, the number of colonies in the C. albicans group treated with CTS-PVA was reduced to a certain extent, showing scattered colonies, but the number of colonies was still countless. The number of all the colonies in the CTS-PVA/PHMG 0.05, CTS-PVA/PHMG 0.1, CTS-PVA/PHMG 0.2, and CTS-PVA/PHMG 0.4 were less than 5 (see Figure 3B).

\section{Characterization}

SEM images of the CTS-PVA/PHMG sponge dressing The SEM images showed that the sponge has an interpenetrating pore structure (see Figure 4). The pore wall surface appeared smooth. Through SEM observation, it was found that the surface pores of the CTS-PVA/PHMG0.05 and CTS-PVA/PHMG0.1 sponge dressings are relatively uniform, with sizes of approximately $50-150 \mu \mathrm{m}$. The CTS-PVA/PHMG0.2 had a large pore size difference, of approximately $20-200 \mu \mathrm{m}$. The CTS-PVA/PHMG0.4 had fewer pores and a compact structure.

\section{Degree of swelling and moisture-retention capacity}

The CTS-PVA/PHMG0.1 sponge was milky white with a soft surface and large number of micropores (Figure 5A). 


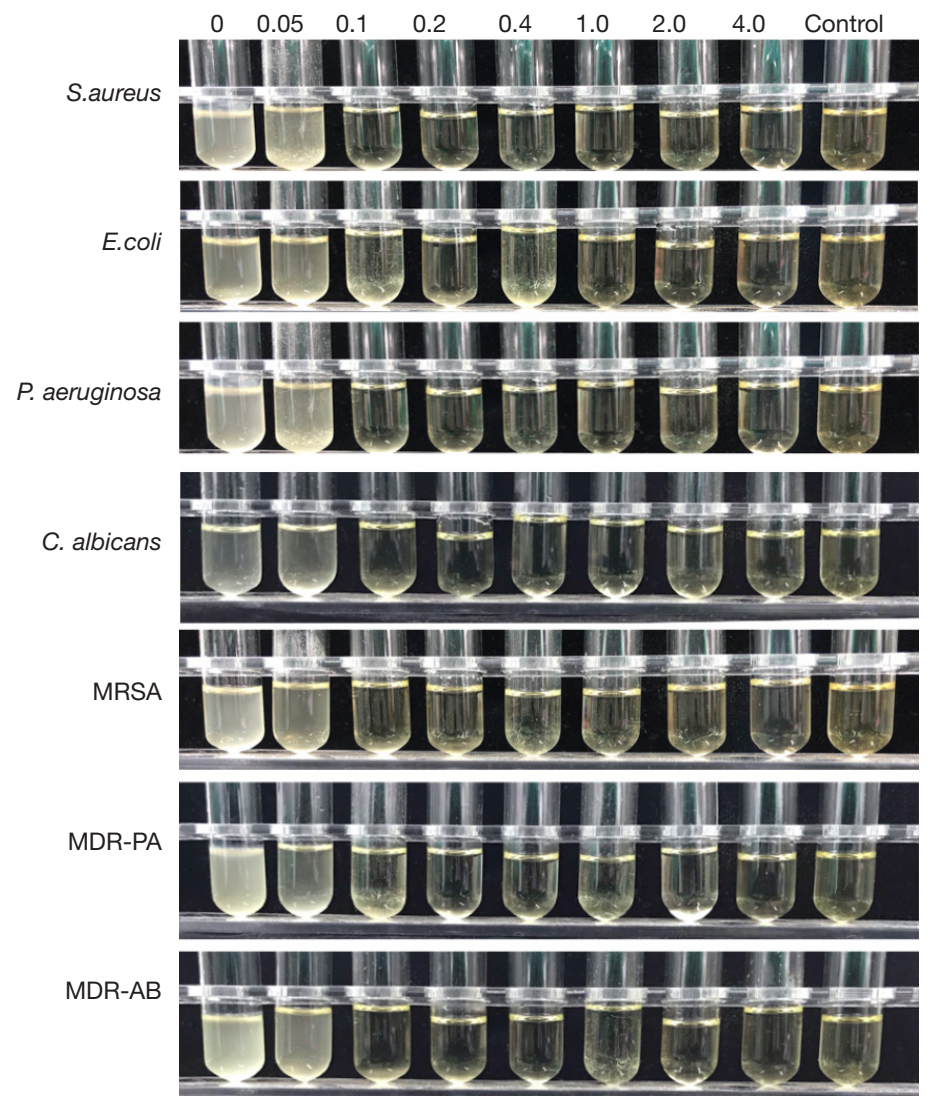

Figure 2 Antibacterial activity of different concentrations of PHMG solution against S. aureus, E. coli, P. aeruginosa, C. albicans, MRSA, MDR-PA, and MDR-AB (wt\%). PHMG, polyhexamethylene guanidine hydrochloride; MRSA, methicillin-resistant Staphylococcus aureus; MDR-PA, multidrug-resistant Pseudomonas aeruginosa; $M D R-A B$, multidrug-resistant Acinetobacter baumannii.

After absorbing water, it appeared as a transparent hydrogel with no collapse (Figure 5B,C).

We analyzed the degree of swelling of the dressings under different simulated physiological conditions. In $1 \mathrm{~h}$, the sponge basically absorbed more than 10 times the weight of UPW, PBS, SBF, and FBS (Figure 5D). The moisture in CTS-PVA/PHMG evaporated slowly, and after $12 \mathrm{~h}$, it retained water close to 20 times the weight of the sponge itself (Figure 5E).

\section{Infectious wound of full-thickness skin defect in mice}

\section{Macroscopic appearances}

On the 3rd day after operation, the wounds treated with the CTS-PVA and Gauze groups showed many inflammatory necrotic and exudate regions. There were no obvious red edges and less exudate observed in the CTS-PVA/PHMG and $\mathrm{Ag}$ groups. On the 7 th day after the operation, the wound in the Gauze group showed a large inflammatory exudate and wound edge swelling with serious wound dressing adhesion. In contrast, the scabs began to fall off, a thin layer of new epithelium grew from the edge of the wound in the CTS-PVA/PHMG groups. On the 10th day after the operation, there were still many inflammatory exudates in the Gauze group. The wound shrinkage of the Ag group was obvious, but the residual wound surface was deeper than that of the CTS-PVA group. In contrast, there was a thin epithelial covering wound in the CTSPVA/PHMG group. On day 14 after the operation, the wound surface of the Gauze group was generally covered by epithelium, and the Ag group was still covered with black scabs. The wounds in the CTS-PVA/PHMG group revealed wound repair within the new epithelium that was significantly superior to that in the other groups (Figure 6).

\section{Wound healing rate}

The wound healing rate of CTS-PVA/PHMG at different healing time points was quantitatively evaluated (see 

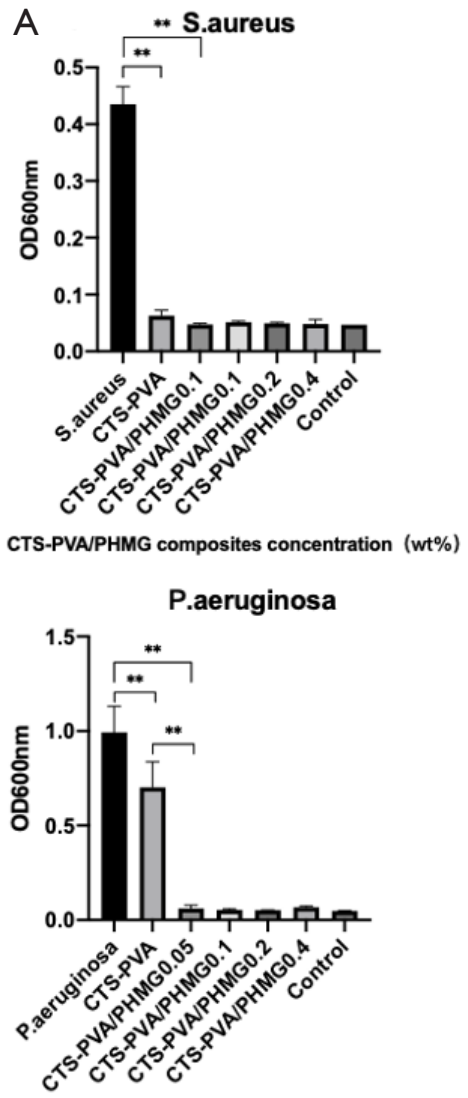

CTS-PVA/PHMG composites concentration (wt\%)

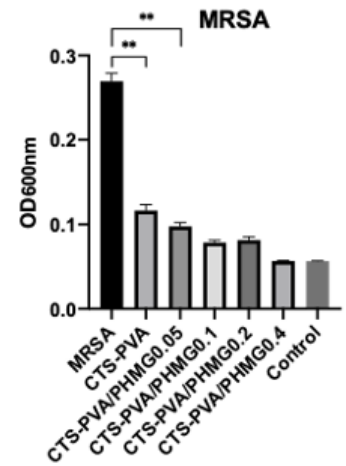

CTS-PVA/PHMG composites concentration (wt\%)

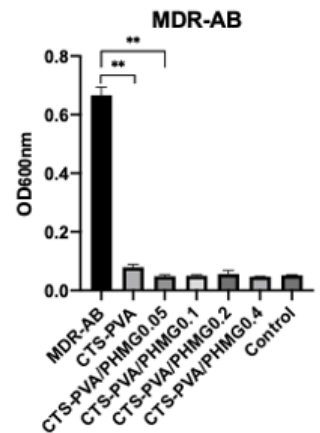

CTS-PVAPPHM composites concentration (wt\%)
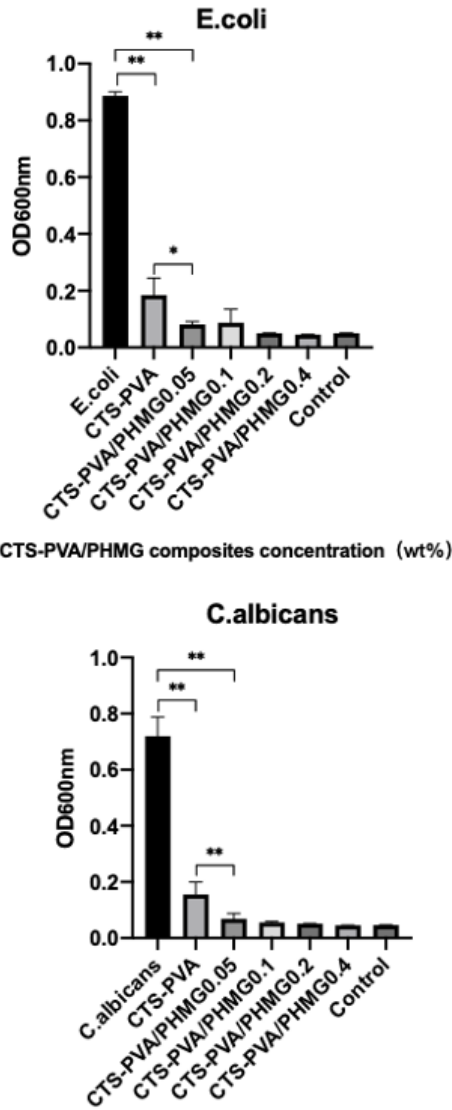

CTS-PVAPHMG composites concentration (wt\%)

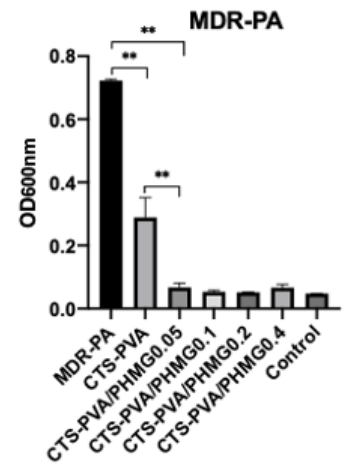

CTS-PVAPHMG composites concentration (wt\%) 
B
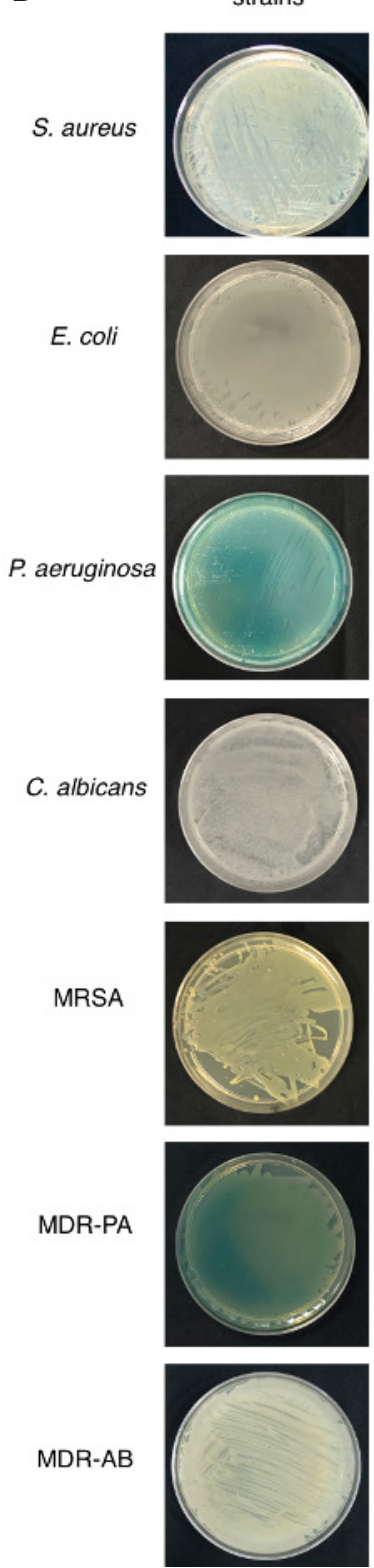

CTS-PVA
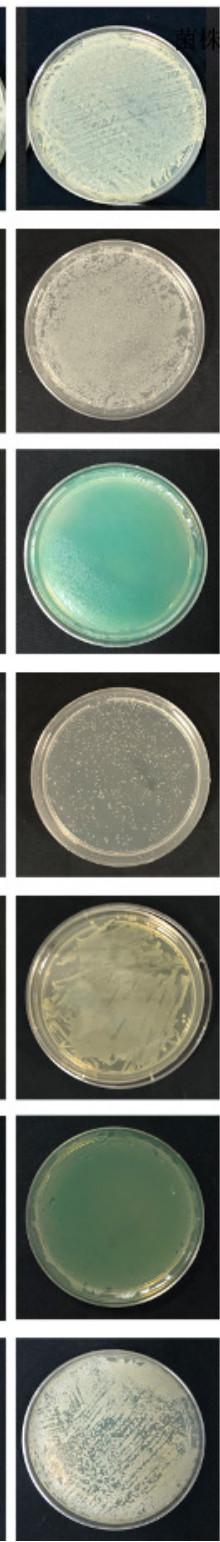

CTS-PVA/PHMG0.05 CTS-PVA/PHMG0.1 CTS-PVA/PHMG0.2 CTS-PVA/PHMG0.4
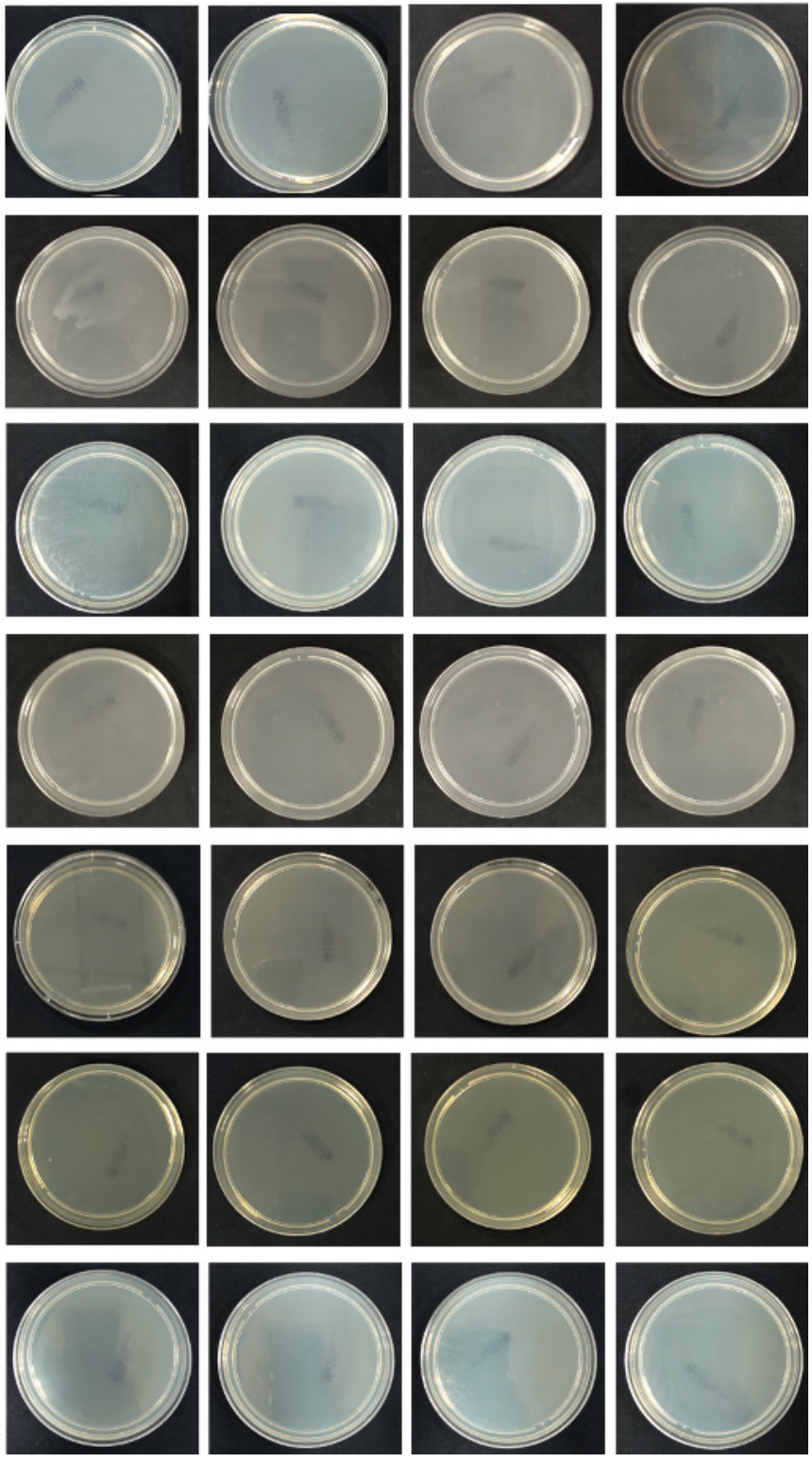

Figure 3 Antibacterial activity of different concentrations of CTS-PVA/PHMG sponges against S. aureus, E. coli, P. aeruginosa, C. albicans, MRSA, MDR-PA, and MDR-AB. (A) OD600 nm of bacterial suspension treated with different concentrations of CTS-PVA/PHMG; *, $\mathrm{P}<0.05$; **, $\mathrm{P}<0.01$. OD, optical density. (B) The bacterial suspensions were plated on $\mathrm{MH}$ plates. The bacterial colonies present on the plates were imaged and counted. CTS, chitosan; PVA, polyvinyl alcohol; PHMG, polyhexamethylene guanidine hydrochloride; MRSA, methicillin-resistant Staphylococcus aureus; MDR-PA, multidrug-resistant Pseudomonas aeruginosa; MDR-AB, multidrug-resistant Acinetobacter baumannii.

Figure 7). On the 3 rd day after operation, the wound healing rate of mice treated with CTS-PVA/PHMG group reached $27.60 \% \pm 0.94 \%$, and the Gauze, CTS-PVA, and $\mathrm{Ag}$ groups were $21.05 \% \pm 2.28 \%, 22.475 \% \pm 3.033 \%$, and
$24.40 \% \pm 1.56 \%$, respectively. The healing rates of the Gauze, CTS-PVA, Ag, and CTS-PVA/PHMG groups reached $32.68 \% \pm 2.20 \%, 39.93 \% \pm 1.66 \%, 43.90 \% \pm 1.24 \%$, and $51.25 \% \pm 4.82 \%$, respectively, on the 7 th day after the 
A

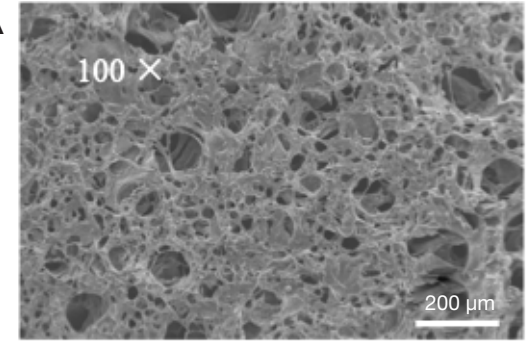

B

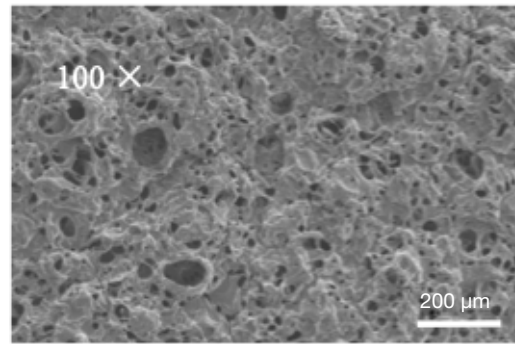

C

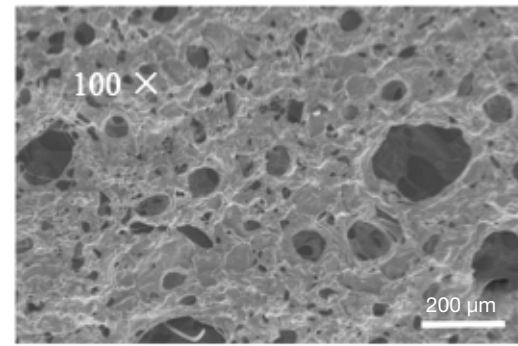

$\mathrm{D}$

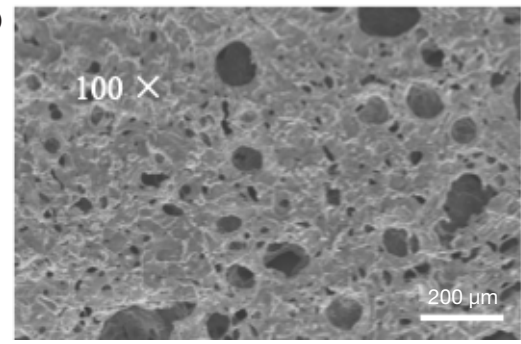

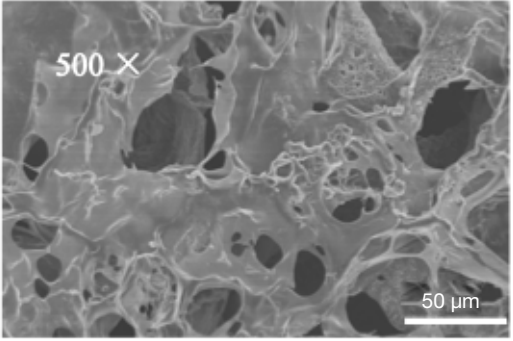


Figure 4 SEM images of CTS-PVA/PHMG surface. (A) CTS-PVA/PHMG 0.05 (B) CTS-PVA/PHMG 0.1 (C) CTS-PVA/PHMG 0.2 (D) CTS-PVA/PHMG 0.4. SE, standard error of the mean; CTS-PVA/PHMG, chitosan-polyvinyl alcohol/ polyhexamethylene guanidine hydrochloride.

operation. On the 10th day after surgery, the healing rate of the CTS-PVA/PHMG group was faster than that of the Gauze group, and the difference was statistically significant $(\mathrm{P}<0.01)$. On the 14th day after surgery, the healing rate of CTS-PVA/PHMG was faster than that of the other groups, and the difference was statistically significant $(\mathrm{P}<0.01)$.

\section{Histological analysis}

On day 3 of H\&E staining, the epidermal skin layer in the various groups was completely defective. Dermal layer inflammatory cell aggregation and tissue necrosis were obvious in the Gauze and CTS-PVA groups. The inflammatory cell infiltrations in the CTS-PVA/PHMG and Ag groups were significantly less than those in the Gauze group. No obvious tissue necrosis was observed in the CTS-PVA/PHMG group. On day 7, many small abscesses were observed in the Gauze group, accompanied by a large numbers of inflammatory cells clustered in the dermis and partial epidermal necrosis. The infection in the CTS-PVA group was less severe than that in the Gauze group, but large numbers of inflammatory cells aggregated together in the dermis. Comparatively speaking, the manifestations 

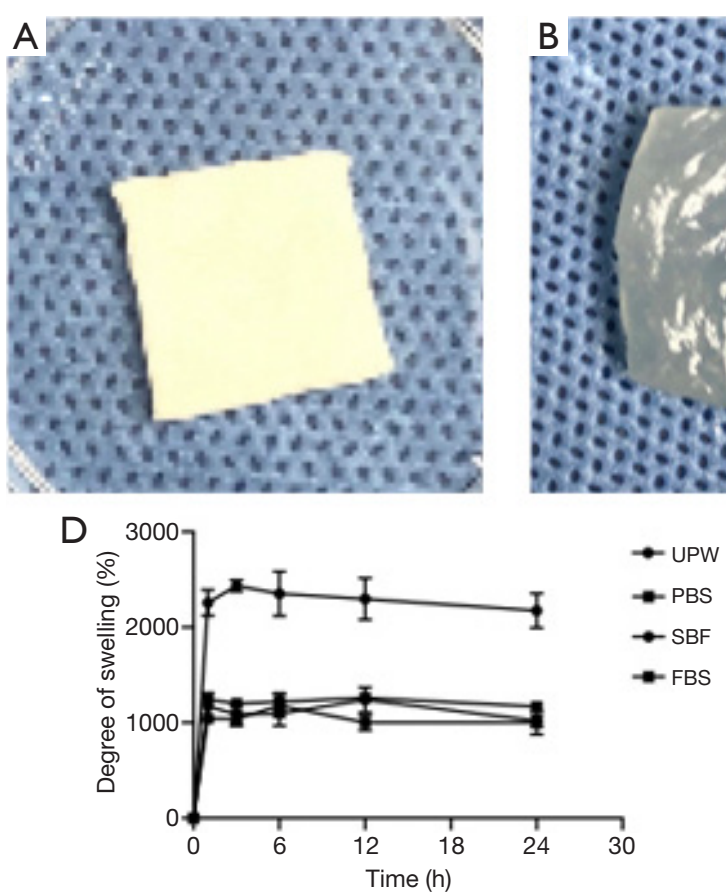
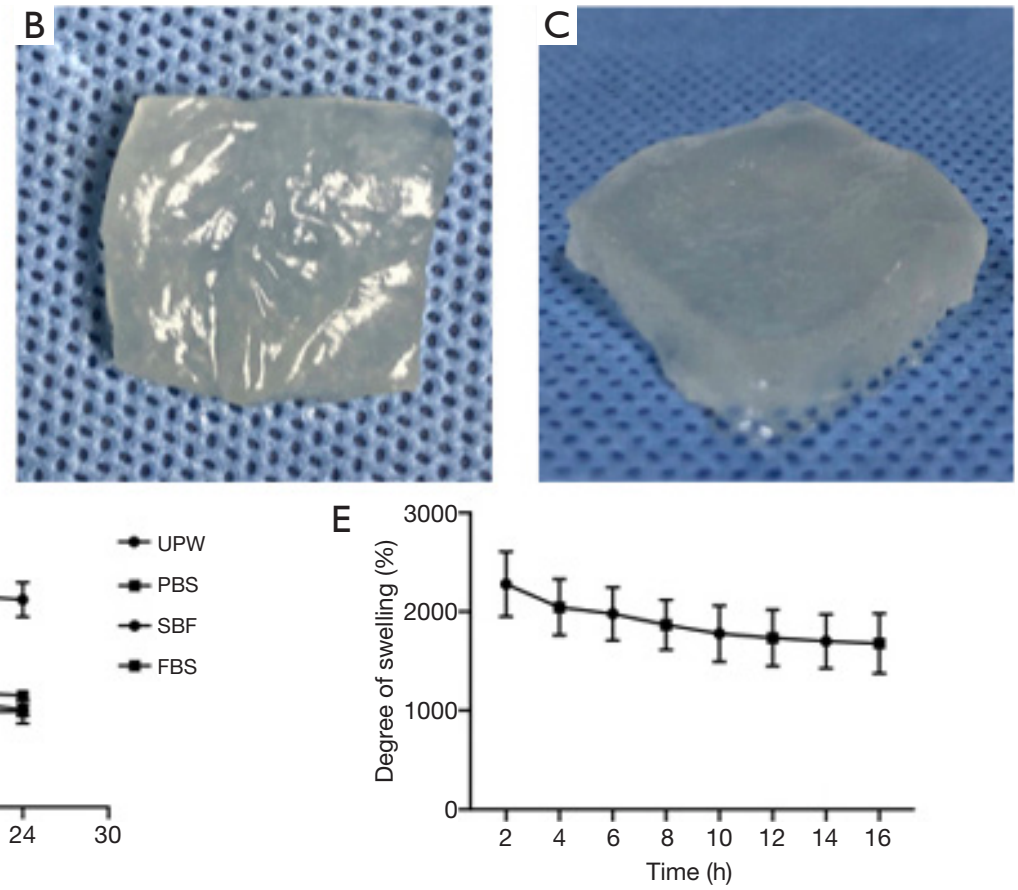

Figure 5 Degree of swelling and moisture-retention capacity. (A) Morphology of dry CTS-PVA/PHMEG0.1 sponge; (B) morphology of the CTS-PVA/PHMG0.1 sponge after UPW absorption; (C) morphology of the CTS-PVA/PHMG0.1 sponge cross section after UPW absorption; (D) swelling degree of CTS-PVA/PHMG0.1 sponges in different media over 24 hours; (E) moisture retention capacity of the CTS-PVA/PHMG0.1 sponge. CTS-PVA/PHMG, chitosan-polyvinyl alcohol/ polyhexamethylene guanidine hydrochloride.

of the Ag group were mild inflammation, with less inflammatory cells scattered infiltration in the granulation tissue, and partial epidermal erosion were still observed. Simultaneously, the wounds in the CTS-PVA/PHMG group displayed scattered infiltration of neutrophils and lymphocytes and a thin re-epithelialized epithelium. On day 14 , the wounds in the Gauze group were covered by a new thin layer of epithelium. The Ag group exhibited scattered hemorrhage between the epithelium and dense granulation tissue. Compared with other groups, the wounds in the CTS-PVA/PHMG group showed basic skin repair and reconstruction (Figure 8).

\section{Discussion}

The skin is the largest and most superficial organ in the entire body (18). The structure and functions of skin are vulnerable to being affected by cuts, burns, and illnesses. An open wound offers a favorable breeding ground for the colonization of microbes (19). An ideal dressing should be capable of protecting the wound surface, maintaining a moist environment, promoting burn wound healing, preventing microbial proliferation, and limiting burn wound progression while minimizing discomfort for patients $(20,21)$.

The main obstacle of antibacterial dressings is the antimicrobial resistance of microorganisms and the toxicity of antibacterial agents to wounds (1). The predominant grampositive organism found in wound infections is Staphylococcus aureus in the initial stage of wound formation (22). Gramnegative pathogens dominate in the initial stage of the infectious process. The most common gram-negative microorganism isolated from wounds is $P$. aeruginosa, followed by E. coli. Candida albicans is the fourth most common cause of burn wound infections overall (23). Emerging multidrug-resistant microorganisms have become more frequent and have caused an unanticipated rise in wound infections, associated sepsis, or death $(22,24)$. In burns and intensive care units, MDR-AB is becoming the main nosocomial infectious bacteria. It is widely resistant to a variety of antibiotics, increasing great difficulties in clinical treatment (25).

We selected PHMG as an antibacterial agent, on account of it being a polycationic bactericidal polymer with broad-spectrum bactericidal properties against 

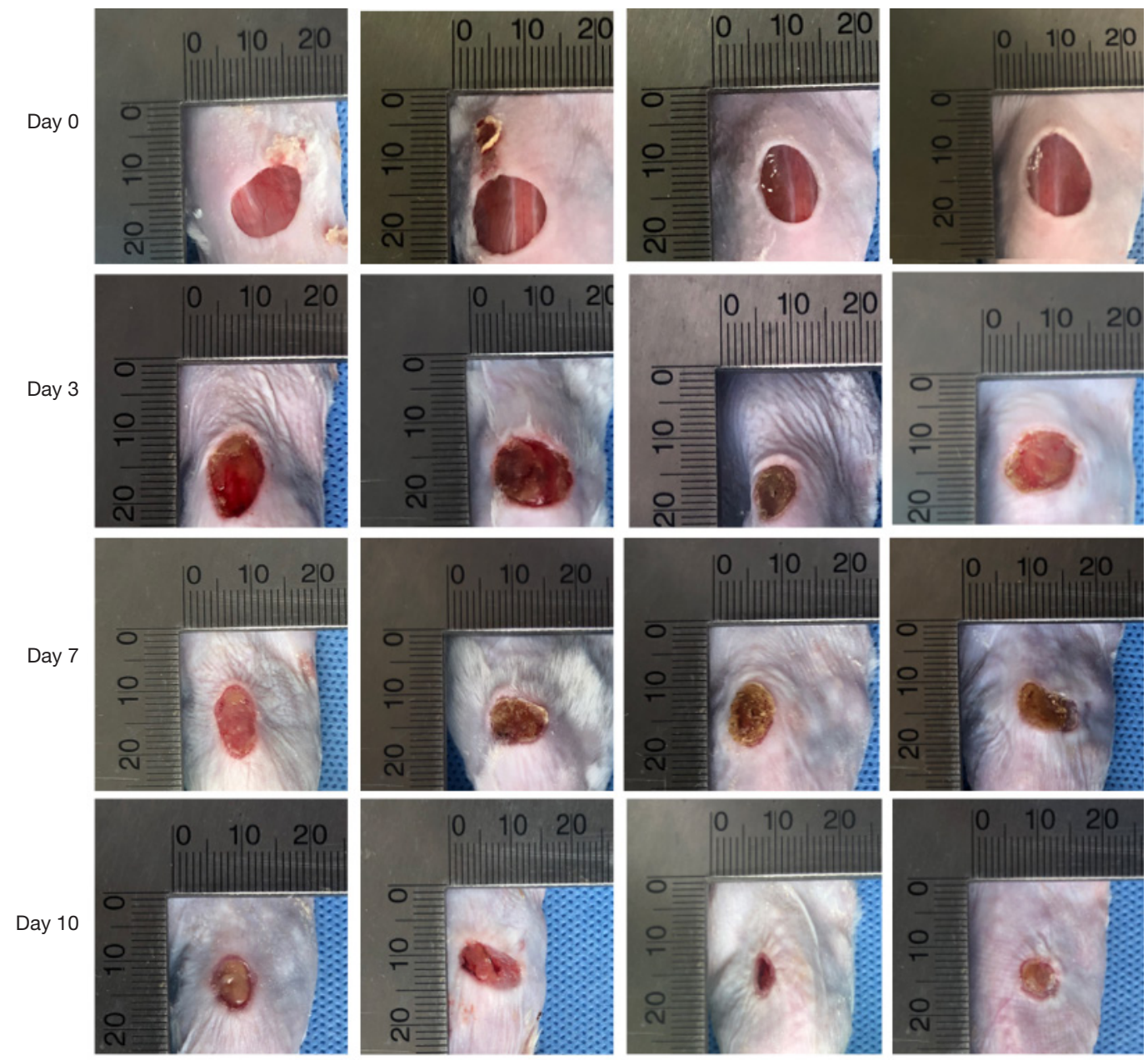

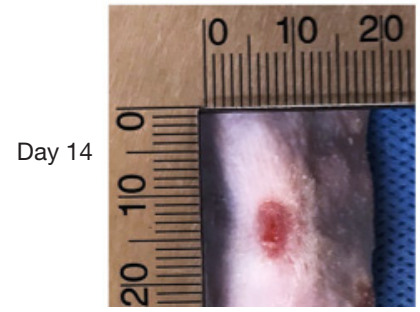

Gauze

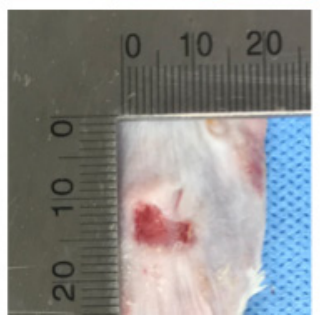

CTS-PVA

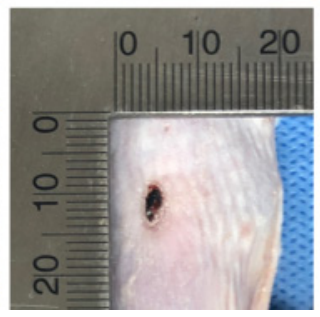

$\mathrm{Ag}$

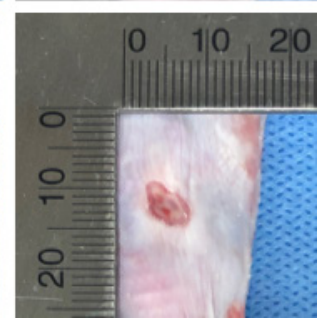

CTS-PVA/PHMG

Figure 6 Representative macroscopic images of full-thickness skin defects in diabetic rats infected with full-thickness skin injury in BALB/ c mice on the 3rd, 7th, 10th, and 14th post-surgical days of the Gauze group, CTS-PVA group, Ag group, and CTS-PVA/PHMG group. CTS-PVA/PHMG, chitosan-polyvinyl alcohol/ polyhexamethylene guanidine hydrochloride.

gram-positive bacteria, gram-negative bacteria, fungi, yeasts, and viruses (26). The PHMG solution displayed high antimicrobial activity against gram-positive bacteria $S$. aureus, gram-negative bacteria E. coli and P. aeruginosa, and fungi $C$. albicans and clinical MDR isolates at a low solution concentration of $0.1 \mathrm{wt}$. We chose another cationic antibacterial polymer, CTS, as the scaffold material loading the antibacterial agent. Due to its strong biocompatibility and antibacterial properties, CTS is widely used in the preparation of dressings; however, pure CTS sponge is brittle (27). Therefore, we choose another kind of polymer, PVA, as the flexible stent of the dressing. A hydrophilic polymer, PVA has excellent biocompatibility and has been applied in various biomedical areas (28). In 
the homogeneous state, PHMG was blended into a CTSPVA sponge scaffold sponge by molecular winding. The antibacterial agent was anchored in the dressing to prevent the antibacterial agent from entering the circulatory system with body fluids, thus avoiding the toxicity and side effects of the antibacterial agent on the human body. The CTSPVA/PHMG sponge prepared by freeze drying avoided residual solvents and toxic molecules during chemical

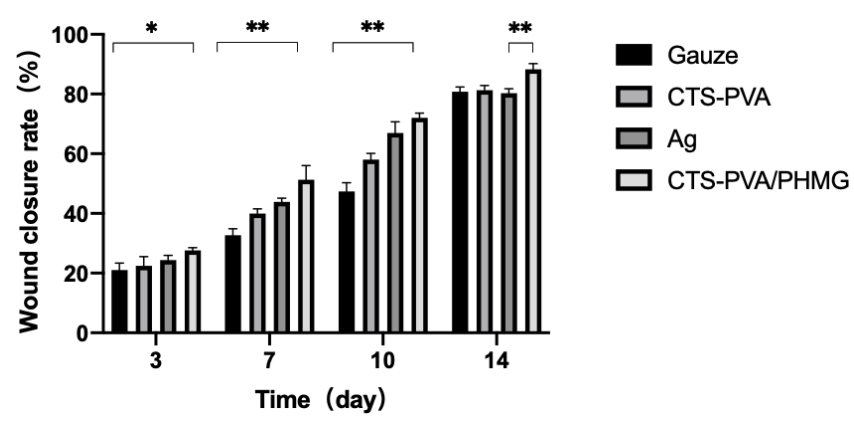

Figure 7 Quantitative analysis of the wound closure rates in each group at the $3 \mathrm{rd}, 7$ th, 10 th, and 14 th days post-surgery $(\mathrm{n}=4$ in each group). *, $\mathrm{P}<0.05 ;{ }^{* *}, \mathrm{P}<0.01$. grafting. Both MIC and MBC in vitro showed that the CTSPVA sponge dressing could suppress the proliferation of strains of E. coli, C. albicans, and S. aureus but did not kill the microorganisms. Therefore, after removing the CTS-PVA dressing, the bacteria still had the ability to multiply. The CTS-PVA/PHMG sponge dressings displayed bactericidal function on laboratory-sensitive strains and a variety of MDR strains at concentrations as low as $0.05 \%$ wt. Both CTS and PHMG are polycationic polymers that kill bacteria due to interactions with the bacterial cell wall (29). The MBC of the PHMG sponge dressing was less than the MIC of the PHMG solution, which indicated that CTS enhanced the electrostatic adsorption of PHMG to strains. The combination of PHMG and CTS displayed a synergistic antibacterial function of polycationic polymers.

The prepared CTS-PVA/PHMG sponge dressing was soft and delicate, which can provide excellent mechanical protection for the wound surface. After absorption of exudate, the CTS-PVA/PHMG sponge dressings became transparent soft gels. Dressing can prevent further injuries and reduce pain. The color change was convenient for observing wound exudation and providing elastic time for


Gauze



CTS-PVA
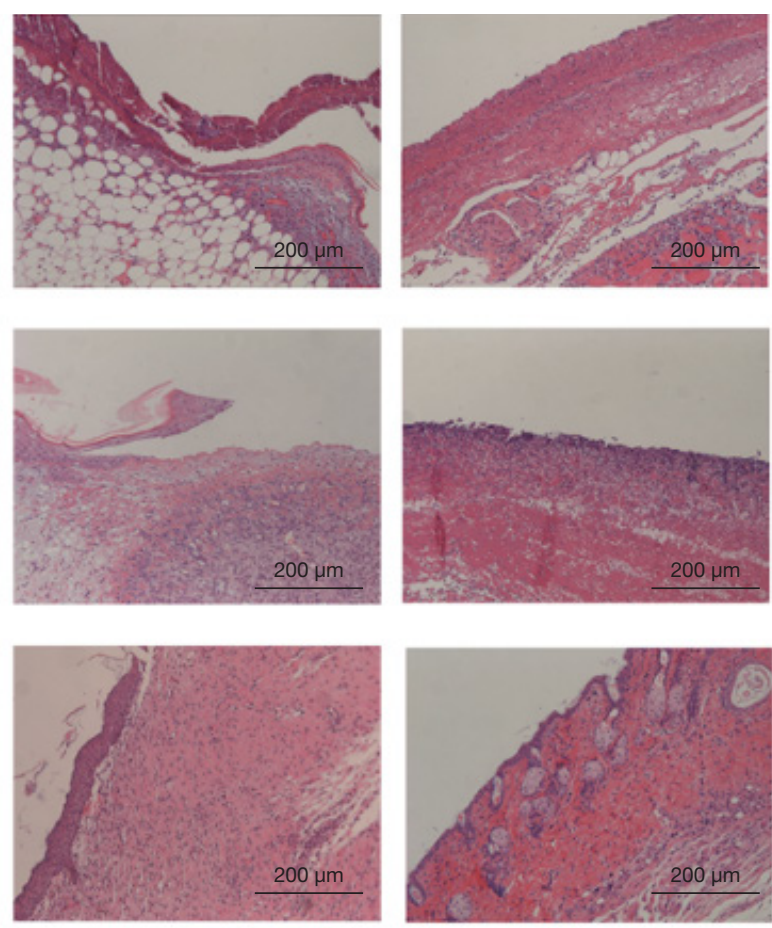

$\mathrm{Ag}$

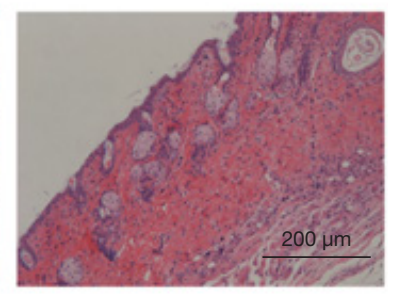

CTS-PVA/PHMG

Figure 8 Representative images of H\&E staining of the wound sections in each group on the 3rd, 7th, and 14th days post-surgery. H\&E, hematoxylin and eosin. 
dressing changes. The SEM showed that the CTS-PVA/ PHMG 0.05 and CTS-PVA/PHMG 0.1 sponge dressings were porous spongy with uniform pores. The porous structure of the dressing was conducive to the penetration of wound exudate, facilitating the transfer of nutrients, and providing the basis for loading biomacromolecule proteins and cells to promote healing. The antibacterial concentration varied against different strains $(30,31)$. Therefore, to enable a wider range of clinical applications of sponge dressings, combined with the results of antibacterial tests and SEM, we finally selected CTS-PVA/PHMG 0.1 as the final concentration of the dressing. The CTSPVA/PHMG0.01 sponge dressing also had high water uptake ability and moisturizing properties. Maintaining a moderately moist environment can promote wound healing and relieve pain during dressings change.

The prepared new CTS-PVA/PHMG sponge dressing was applied to the model of $P$. aeruginosa infectious wounds on the backs of mice. The Ag group and CTS-PVA/PHMG group were both effective in the early healing of infected wounds in mice, indicating that CTS-PVA/PHMG can significantly control wound infection. In the late stage of healing, the general situation of the wounds indicated that the CTS-PVA/PHMG sponge dressing had a better healing effect on skin tissue than the Ag group. This may be because a relative release of silver nanoparticles (AgNPs) stimulated the wound and affected wound healing. The healing speed and quality of CTS-PVA/PHMG sponges were significantly superior to those of common dressings, indicating that the dressing not only provided mechanical protection for the wound but also avoided secondary injury to the wound. A dressing with a strong water uptake ability can reduce bacterial growth. The H\&E staining showed that CTSPVA/PHMG significantly reduced wound inflammation. After absorbing wound exudate, the moist environment provided by the sponge was also more conducive to the formation of wound skin without epithelial avulsion and destruction of granulation tissue during dressing changes, thereby increasing the speed of epithelialization and promoting healing. Further, we will continue to explore the potential of CTS-PVA/PHMG loading biomacromolecule proteins and cells to have an even greater impact on emerging wound applications.

\section{Conclusions}

In conclusion, we successfully prepared and characterized a new CTS-PVA/PHMG broad-spectrum antibacterial dressing using the freeze-drying method that can be beneficial for wound healing. These findings provide a novel application method for infectious wound skin repair.

\section{Acknowledgments}

Funding: This work was supported by four research projects of Major Project of Military Logistical Support Department (AWS15J003, ALB19J001), National Natural Science Foundation of China (81571894), (81772067).

\section{Footnote}

Reporting Checklist: The authors have completed the ARRIVE reporting checklist. Available at http://dx.doi. org/10.21037/atm-21-509

Data Sharing Statement: Available at http://dx.doi. org/10.21037/atm-21-509

Conflicts of Interest: All authors have completed the ICMJE uniform disclosure form (available at http://dx.doi. org/10.21037/atm-21-509). The authors have no conflicts of interest to declare.

Ethical Statement: The authors are accountable for all aspects of the work in ensuring that questions related to the accuracy or integrity of any part of the work are appropriately investigated and resolved. This study was performed in accordance with the Guide for Care and Use of Laboratory Animals of National Research Council and approved by the Fourth Medical Center Affiliated to PLA General Hospital, Beijing, China.

Open Access Statement: This is an Open Access article distributed in accordance with the Creative Commons Attribution-NonCommercial-NoDerivs 4.0 International License (CC BY-NC-ND 4.0), which permits the noncommercial replication and distribution of the article with the strict proviso that no changes or edits are made and the original work is properly cited (including links to both the formal publication through the relevant DOI and the license). See: https://creativecommons.org/licenses/by-nc-nd/4.0/.

\section{References}

1. Simões D, Miguel SP, Ribeiro MP, et al. Recent advances on antimicrobial wound dressing: A review. Eur J Pharm 
Biopharm 2018;127:130-41.

2. Burnham JP, Kirby JP, Kollef MH. Diagnosis and management of skin and soft tissue infections in the intensive care unit: a review. Intensive Care Med 2016;42:1899-911.

3. Cardona AF, Wilson SE. Skin and soft-tissue infections: a critical review and the role of telavancin in their treatment. Clin Infect Dis 2015;61 Suppl 2:S69-78.

4. Ki V, Rotstein C. Bacterial skin and soft tissue infections in adults: A review of their epidemiology, pathogenesis, diagnosis, treatment and site of care Can J Infect Dis Med Microbiol 2008;19:173-84.

5. Rudramurthy GR, Swamy MK, Sinniah UR, et al. Nanoparticles: alternatives against drug-resistant pathogenic microbes. Molecules 2016;21:836.

6. Wasiak J, Cleland H, Campbell F, et al. Dressings for superficial and partial thickness burns. Cochrane Database Syst Rev 2013;2013:CD002106.

7. Nimal TR, Baranwal G, Bavya MC, et al. Antistaphylococcal Activity of Injectable Nano Tigecycline/ Chitosan-PRP Composite Hydrogel Using Drosophila melanogaster Model for Infectious Wounds. ACS Appl Mater Interfaces 2016;8:22074-83.

8. Contardi M, Heredia-Guerrero JA, Perotto G, et al. Transparent ciprofloxacin-povidone antibiotic films and nanofiber mats as potential skin and wound care dressings. Eur J Pharm Sci 2017;104:133-44.

9. Monteiro N, Martins M, Martins A, et al. Antibacterial activity of chitosan nanofiber meshes with liposomes immobilized releasing gentamicin. Acta Biomater 2015;18:196-205.

10. Marin S, Vlasceanu GM, Tiplea RE, et al. Applications and toxicity of silver nanoparticles: a recent review. Curr Top Med Chem 2015;15:1596-604.

11. Negut I, Grumezescu V, Grumezescu AM. Treatment Strategies for Infected Wounds. Molecules 2018;23:2392.

12. Yang $Y$, Qin Z, Zeng W, et al. Toxicity assessment of nanoparticles in various systems and organs. Nanotechnol Rev 2016;6:2191-7.

13. Timofeeva L, Kleshcheva N. Antimicrobial polymers: mechanism of action, factors of activity, and applications. Appl Microbiol Biotechnol 2011;89:475-92.

14. Sun S, An Q, Li X, et al. Synergistic effects of chitosanguanidine complexes on enhancing antimicrobial activity and wet-strength of paper. Bioresour Technol 2010;101:5693-700.

15. Li Y, Tian Y, Zheng W, et al. Composites of Bacterial Cellulose and Small Molecule-Decorated Gold
Nanoparticles for Treating Gram-Negative BacteriaInfected Wounds. Small 2017;13(27). doi: 10.1002/ smll.201700130.

16. Wang Y, Xue X, Yang H, et al. Preparation and characterization of $\mathrm{Zn} / \mathrm{Ce} / \mathrm{SO} 4$ 2-doped titania nanomaterials with antibacterial activity. Appl Surf Sci 2014;292:608-14.

17. Shi Q, Qian Z, Liu D, et al. GMSC-Derived Exosomes Combined with a Chitosan/Silk Hydrogel Sponge Accelerates Wound Healing in a Diabetic Rat Skin Defect Model. Front Physiol 2017;8:904.

18. Khavkin J, Ellis DA. Aging skin: histology, physiology, and pathology. Facial Plast Surg Clin North Am 2011;19:22934.

19. Ramakrishnan K, Salinas RC, Agudelo Higuita NI. Skin and Soft Tissue Infections. Am Fam Physician 2015;92:474-83.

20. Yoshino Y, Ohtsuka M, Kawaguchi M, et al. The wound/ burn guidelines - 6: Guidelines for the management of burns. J Dermatol 2016;43:989-1010.

21. International Consensus. Acellular matrices for the treatment of wounds. An expert working group review. Wounds International 2010. Available online: https:// www.woundsinternational.com/resources/details/acellularmatrices-treatment-wounds

22. Mauldin PD, Salgado CD, Durkalski VL, et al. Nosocomial infections due to methicillin-resistant Staphylococcus aureus and vancomycin-resistant enterococcus: relationships with antibiotic use and cost drivers. Ann Pharmacother 2008;42:317-26.

23. Azzopardi EA, Azzopardi SM, Boyce DE, et al. Emerging gram-negative infections in burn wounds. J Burn Care Res 2011;32:570-6.

24. Wibbenmeyer LA, Kealey GP, Latenser BA, et al. Emergence of the USA300 strain of methicillin-resistant Staphylococcus aureus in a burn-trauma unit. J Burn Care Res 2008;29:790-7.

25. Branski LK, Al-Mousawi A, Rivero H, et al. Emerging infections in burns. Surg Infect (Larchmt) 2009;10:389-97.

26. Müller G, Kramer A. Effect of selected wound antiseptics on adult articular cartilage (bovine sesamoid bone) in the presence of Escherichia coli and Staphylococcus aureus. J Orthop Res 2005;23:127-33.

27. Qian Z, Bai Y, Zhou J, et al. A moisturizing chitosansilk fibroin dressing with silver nanoparticles-adsorbed exosomes for repairing infected wounds. J Mater Chem B 2020;8:7197-212.

28. Kamoun EA, Kenawy ES, Chen X. A review on polymeric 
hydrogel membranes for wound dressing applications:

PVA-based hydrogel dressings. J Adv Res 2017;8:217-33.

29. Gilbert P, Moore LE. Cationic antiseptics: diversity of action under a common epithet. J Appl Microbiol 2005;99:703-15.

30. Oulé MK, Azinwi R, Bernier AM, et al. Polyhexamethylene guanidine hydrochloride-based disinfectant: a novel tool to fight meticillin-resistant Staphylococcus aureus and

Cite this article as: Yue X, Liu L, Wu Y, Liu X, Li S, Zhang Z, Han S, Wang X, Chang Y, Bai H, Chai J, Hu S, Wang H. Preparation and evaluation of chitosan-polyvinyl alcohol/polyhexamethylene guanidine hydrochloride antibacterial dressing to accelerate wound healing for infectious skin repair. Ann Transl Med 2021;9(6):482. doi: 10.21037/atm21-509 nosocomial infections. J Med Microbiol 2008;57:1523-8.

31. Mattheis C, Wang H, Meister C, et al. Effect of guanidinylation on the properties of poly(2aminoethylmethacrylate)-based antibacterial materials. Macromol Biosci 2013;13:242-55.

(English Language Editor: J. Jones) 